\title{
Génesis y estética en la lírica chaceliana: transmutación de significantes y clasicismo apolíneo en "Apolo"
}

Genesis and aesthetics in the chacelian lyric: transmutation of signifiers and Apollonian classicism in "Apollo"

Endika Basáñez Barrio

${ }^{1}$ Departamento de Filología Hispánica, Románica y Teoría de la Literatura, Universidad del País Vasco UPV/EHU, España (endika.basanez@ehu.eus)

Recibido el 15 de junio de 2017; revisado el 27 de junio de 2017; aceptado el 15 de julio de 2017; publicado el 01 de septiembre de 2017

RESUMEN: El presente artículo se propone como objetivo principal revisar uno de los poemas más célebres de la autora vallisoletana Rosa Chacel, "Apolo", focalizando dicho análisis en la vinculación existente entre la génesis y la estética del mismo a través de una hipótesis basada en la trasmutación de los materiales artísticos heterogéneos a la que la autora da lugar en el proceso de la incorporación de la escultura del dios griego Apolo en su poema, que no el dios en sí, como ha apuntado hasta la fecha gran parte de la crítica literaria.

PALABRAS CLAVE: Literatura, poesía española, lírica, Rosa Chacel.

ABSTRACT: The main aim this paper tries to reach is to review Spanish female writer Rosa Chacel's lyrical poem "Apolo" in order to analyze the way she finds to incorporate Apollo Greek god's sculpture into her piece of work, which comes to add a new point of view when reading the poem itself because, so far, it has just been said that Chacel uses Greek god's characteristics to write her piece of art and not his sculpture, that is the idea this paper defends.

KEYWORDS: Literature, Spanish poetry, lyrical poetry, Rosa Chacel. 
Ciertamente la autora española Rosa Chacel Arimón (Valladolid, 1898-Madrid, 1994), más conocida como -únicamente- Rosa Chacel, presenta una extensa producción narrativa1 frente a tan solo -y literalmente- un par de obras de génesis lírica, por lo que la escritora está fundamentalmente vinculada al texto narrativo, tal y como lo demuestra la cantidad de publicaciones y bibliografía que puede hallarse en cuanto a la investigación llevada a cabo hasta la fecha en lo referente a su producción artística, frente a las escasas anotaciones e impresiones editoriales relacionadas con su poesía. De cualquier modo, esta última bien puede resumirse en dos principales obras que se corresponden con $A$ la orilla de un pozo (1936) y Versos prohibidos (1978), recogidas ambas, a su vez, en Poesía (19311991), publicada en Barcelona por la editorial Tusquets en su célebre colección Nuevos Textos Sagrados, en el año 1992.

Lo cierto es que uno de los aspectos más interesantes de la producción lírica de la autora vallisoletana reside en la estética que envuelve al contenido de sus poemas y, a su vez, actúa como cimiento de los mismos, es decir, estética y génesis -o viceversa- están íntimamente ligadas; la estética de la lírica chaceliana (y su génesis) es, por lo tanto, el tema sobre el que pretendemos profundizar a lo largo de las páginas que conforman el presente artículo, tomando como ejemplo para ello el caso de uno de sus poemas más recordados por la comunidad hispanista, "Apolo" donde, además, el vínculo entre creación y estética se ve materializado a través de la transmutación de contenidos artísticos y, de forma más concreta, entre la escultura del dios griego Apolo que la misma presenció en su juventud y su forma de incorporar la figura (y no el mito) en su poema. De esta forma, pretendemos dar lugar a un nuevo análisis de la lírica chaceliana a la vez que reavivamos el estado de la cuestión en cuanto al estudio del poema mencionado por la crítica literaria, ofreciendo nuevas posibilidades de aproximación al mismo.

No cabe duda alguna que la construcción del poema, en su estructura más superficial, se fundamenta sobre la deidad griega correspondiente al mito de Apolo, dios de características vinculadas a la belleza física y a la perfecta mesura de esta beldad, entre muchas otras dotadas de connotaciones manifiestamente positivas:

Apolo es un dios que pertenece a la segunda generación de los Olímpicos. Es hijo de Zeus y Leto y hermano de la diosa Ártemis [...]. Se representaba a Apolo como un dios muy hermoso, alto, notable especialmente por sus largos bucles negros de reflejos azulados, como los pétalos

\footnotetext{
${ }^{1}$ No en vano, son muchas las ocasiones en las que nos hemos topado con el apelativo de "gran prosista de la Generación del 27", alabando así su extensa composición narrativa en prosa, especialmente evidente en el apartado correspondiente a
} 
del pensamiento. No es de extrañar que tuviese numerosos amoríos con Ninfas y con mortales (Grimal, 2008, 35-36).

Lo cierto es que la elección del mito griego muestra un gran acervo cultural por parte de la autora en tanto que, dentro del abanico de posibilidades temático-estéticas disponibles para la construcción del poema, Chacel opta por mostrar una actitud intelectual, ya que fundamenta la génesis y la estética del poema sobre un mito clasicista (actitud que, por otro lado, se repite en gran parte de su producción lírica, tal y como ocurre también en los poemas - de nombres parlantes per se- "Narciso", "Anticoo" o "La Reina Artemisa"). Anecdóticamente, la autora, en su faceta como escritora de poemas, actúa en el panorama literario de la Transición Española como eslabón de una serie de autoras coetáneas, de corte fundamentalmente feminista, que nutren su producción lírica en temas clasicistas, por lo que la estética de estos manifiesta una actitud ciertamente culturalista, plagada de, valga la redundancia, referencias culturales; tal es el caso de la única poetisa perteneciente al grupo introductor de la posmodernidad lírica en España, los novísimos2, Ana María Moix, o, ya en la década de los años 80, Ana Rossetti a través de su celebrada y premiada publicación Los devaneos de Erato (1980), si bien con transgresoras propuestas de remodelación estética a través del erotismo y el sexo (entendido, además, en todas sus formas de práctica posibles). No obstante, pese a que la producción literaria de la autora vallisoletana podría ser considerada como femenina en tanto que, evidentemente, la autora es mujer, en ningún caso, debe asociarse el término "producción femenina" como sinónimo de "feminismo" ya que ésta no mostró actitudes literarias explícitas que pudieran ser identificadas con tal movimiento, llegando incluso a negar en lo personal la disociación entre lo típicamente masculino y femenino; por lo tanto, no existe para la autora necesidad ideológica de la defensa de la cuestión femenina:

Rosa Chacel rechaza por completo la creencia en unas esencias que conduzcan a la completa diferenciación y segregación de los sexos. Se resiste a creer que las diferencias, que sin duda se dan entre los sexos, sean tan fundamentales como para construir la razón misma de lo masculino y lo femenino como entes separados. Menos aún acepta la identificación de lo humano con lo varonil y de lo masculino con la cultura [...] Rosa Chacel considera que todos los seres humanos tienen las mismas posibilidades, siendo el conocimiento, la inteligencia y la cultura los factores que igualarían a ambos sexos [...] (Osborne, 1987, pp. 97-112).

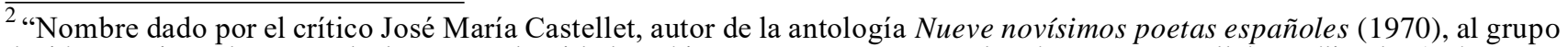
de jóvenes introductores de la posmodernidad poética en España: Manuel Vázquez Montalbán, Félix de Azúa, Pere Gimferrer, Ana María Moix, Vicente Molina Foix, Leopoldo María Panero, Guillermo Carnero, José María Álvarez y Antonio Martínez Carrión. Los 'novísimos' pusieron término a la tradición poética vigente desde los años cincuenta. Metapoesía, metalenguaje, culturalismo y vitalismo son rasgos propios del grupo" (Quesada Marco, 1997, p. 341).
} 
De cualquier modo, el clasicismo estético del poema chaceliano, en una estructura más profunda, abandona ya el dios Apolo mantenido en el acervo común para fundamentarse sobre un proceso de transmutación de contenidos artísticos en tanto que el poema se recrea en las características físicas de la escultura del mito que la autora decide convertir en palabra lírica y, por tanto, literatura. No en vano, la propia autora alude de forma explícita a la transmutación de dichos contenidos en su autobiografía al apuntar que "En mi emoción ante la escultura griega predominaba un sentimiento de facilidad, de comodidad por afinidad. La forma así, tal como la veía en ella no tenía secreto para mí. [...] La escultura era la forma puesta ahí, y yo podía dar vueltas alrededor [...] La visión del Apolo en el rincón oscuro -veinte años después traté de fijarla en un poemaz [...]”. A lo que en pleno éxtasis del recuerdo de la visión de la escultura del dios griego añade: "El caso es que presidió y presidirá mi vida todo lo que dure- La contemplación del Apolo fue como la adquisición de todo el saber; [...]" (-ambas citas2004, p. 166). Así pues, si aplicamos a este estudio la distinción saussurreana del significado y el significante para la comprensión del signo lingüístico, la autora se nutre del mismo significado (Apolo), pero opta por desechar la representación escultural a favor de la lírico-literaria, es decir, opta por el significante literario (palabra), frente al significante artístico correspondiente a la forma escultural.

\begin{tabular}{|c|c|c|}
\hline$\underline{\text { Significante }}$ & Significado & Significante \\
\hline Escultura & Apolo & Palabra lírica \\
\hline \multicolumn{3}{|c|}{ Transmutación de significantes } \\
\hline
\end{tabular}

Si preferimos hacer referencia al código semiótico en cuanto a la transmutación de significantes, nos hallamos en la misma situación ya anotada: la autora pretende emitir las mismas peculiaridades que presenta la forma escultural de Apolo, pero se ve obligada a emplear la palabra como signo en lugar del material propio de la escultura; en cualquier caso, el mensaje que se transmite en los diferentes medios (escultura o poema) es el mismo, solo que cada uno se adecúa a las particularidades de su medio de expresión (escultura artística frente a creación literaria)

\footnotetext{
${ }^{3}$ Precisamente dicho fragmento es el que nos reveló la posibilidad de abrir un camino de análisis entre génesis y estética del poema a través de la incorporación de la escultura del Apolo que la vallisoletana presenció en palabra lírica, es decir, la transmutación de los materiales artísticos.
} 


\begin{tabular}{|c|c|}
\hline Poema & Análisis \\
\hline $\begin{array}{l}\text { Habitante de los anchos portales } 4 \\
\text { dondes el laurel de la sombra oculta el arpa de la araña, }\end{array}$ & $\begin{array}{l}\text { Introducción de la escultura } \\
\text { en la lírica (desplazamiento } \\
\text { del "ancho portal" al } \\
\text { poema). }\end{array}$ \\
\hline $\begin{array}{l}\text { ¡El silencio dictado por tu mano, } \\
\text { la línea entre tus labios sostenida, } \\
\text { tu suprema nariz exhalando un aliento } \\
\text { por gemelas vertientes recorriendo los valles de tu pecho, } \\
\text { y en torno a tus tobillos un espacio }\end{array}$ & $\begin{array}{l}\text { Recreación en las } \\
\text { características físicas de la } \\
\text { figura de Apolo, no del } \\
\text { mito. }\end{array}$ \\
\hline $\begin{array}{l}\text { ¡Eterna, eternamente un universo a imagen tuya! } \\
\text { Con la frente a la altura de tu plinto, }\end{array}$ & $\begin{array}{l}\text { Alusión a la imagen y al } \\
\text { sustento de la escultura (el } \\
\text { plinto). }\end{array}$ \\
\hline El trazo de tu norma, en el basalto & $\begin{array}{l}\text { Mención al basalto como } \\
\text { material físico (el trazo } \\
\text { apolíneo lo modela). }\end{array}$ \\
\hline $\begin{array}{l}\text { Sobre mí, solo eterno } \\
\text { tu mandato de luz, Verdad y Forma }\end{array}$ & $\begin{array}{l}\text { Forma como propiedad } \\
\text { física (mandato a partir de la } \\
\text { forma). }\end{array}$ \\
\hline
\end{tabular}

En efecto, la autora castellano-leonesa había ya mostrado desde su adolescencia un especial interés por las artes plásticas, especialmente la pintura y la escultura, formas estas que resultarían ser esperables de hallar en su obra literaria como herencia o rastros de su aproximación más temprana al

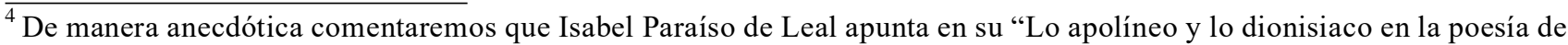
Rosa Chacel" que este primer verso de "Apolo" evidencia el rasgo vanguardista del poema en tanto que la autora trata al mito de "manera insólita" (y para ello recurre a este primer verso: "Habitante de los anchos portales"). Nosotros no nos mostramos de acuerdo con su postura en tanto que creemos que Paraíso de Leal no comparte la recuperación del mito a partir de la escultura del mismo (tesis que intentamos defender en este artículo), con lo cual hablar de él como habitante de los anchos portales supondría la introducción en el poema de la escultura del Apolo del Ateneo (y no del mito, abstracto). Además, creemos que el poema, en suma, no refleja el Vanguardismo que sí presenta (y de manera inequívocamente explícita) otros poemas chacelianos, como es el caso, por ejemplo, de "Ausencia". Es por eso por lo que hemos decidido seleccionar "Apolo" como poema de estética culturalista-clasicista sin la primacía de la influencia vanguardista (al igual que ocurre en "Anticoo" o "La Reina Artemisa"). PARAÍSO DE LEAL, Isabel (1994), "Lo apolíneo y lo dionisiaco en la poesía de Rosa Chacel” en LATRE, María Pilar Martínez (ed.), Actas del congreso en homenaje a Rosa Chacel: ponencias y comunicaciones. Universidad de La Rioja: Logroño, pág. 43.

${ }^{5}$ El subrayado de los diversos elementos en los versos de Rosa Chacel a lo largo de todo el artículo es de nuestra autoría para hacer énfasis así en nuestra exposición.
} 
mundo del arte, lo que vendría a avalar nuestra tesis en cuanto a la transmutación de significantes artísticos:

De niña, de joven, iba para artista plástica, primero en academias vallisoletanas de dibujo y pintura, y por otros centros madrileños después, hasta desembocar en la escultura y en la Escuela de Bellas Artes de San Fernando. Uno de sus libros, Desde el amanecer (1972) relata precisamente su niñez en Valladolid -su extraño y precocísimo aprendizaje de la palabra a los cinco meses, o de lectura a los tres años, de dónde si no saldría su extraña relación con una de las prosas más asombrosas de nuestra historia- [...] Pero el dibujo, la pintura y la escultura dejaron paso a una nueva vocación, la de la escritura, donde toda esa capacidad visual se plasmaría en unas extrañas geografías interiores de una misteriosa plasticidad, que se infiltró primero por el sendero de la escritura. Gran lectora desde su infancia, Rosa Chacel se introdujo desde el principio, en sus años de estudiante de artes plásticas, en los círculos culturales, intelectuales y literarios de la capital (Conte, 1993, pp. 22).

De este modo resulta ahora más explícito anotar que el origen del poema "Apolo" se halla ya, como es evidente, en el mito clásico, sólo que la autora lo recoge a partir de su manifestación escultural, que ella transforma después en lírica: origen y estética se hallan, pues, íntimamente ligados en el poema que nos ocupa en tanto que este surge de la escultura y se recrea (y crea con ello el poema) a partir de las cualidades apolíneas de la escultura del mito homónimo:

$$
\begin{gathered}
\text { Génesis del poema } \\
\text { Apolo (mito) } \longrightarrow \text { Apolo (escultura) }
\end{gathered}
$$

Estética del poema

Apolo (lírico)

Ciertamente, la estética del poema analizado presenta un interesante rasgo en su composición que denota el astuto y sabio manejo de Chacel en su creación lírica, y es que el poema no solo se construye sobre la figura del dios Apolo y sus características físicas, sino que se alimenta, de igual modo, de un eje compositivo apolíneo a través de dos medios: el lingüístico, más explícito, y el orden estrófico que lo construye. En cuanto a los rasgos verbales, la autora selecciona varios sintagmas que aluden al orden, la belleza y la propia norma de la beldad de manera evidente, con lo cual el eje apolíneo lingüístico sobre el que se construye el poema queda explicitado a nivel lingüístico (fundamentalmente, a través del campo semántico formado por partes de la escultura como son los rasgos físicos y el material que le da sustento): 
¡El silencio dictado por tu mano,

la línea entre tus labios sostenida,

tu suprema nariz exhalando un aliento

como brisa en las praderas,

por gemelas vertientes recorriendo los valles de tu pecho,

y en torno a tus tobillos un espacio

pálido como el alba!

¡Eterna, eternamente un universo a imagen tuya!

Con la frente a la altura de tu plinto,

El trazo de tu norma, en el basalto

Sobre mí, solo eterno

tu mandato de luz, Verdad y Forma.

De igual forma, la distribución estrófica se presenta de manera encadenada en un orden gradual en cuanto a la expresión de las características del dios griego, incidiendo, en su conjunto, en la eterna belleza que reside en la mesura de lo apolíneo; cada estrofa viene, pues, a actuar como un eslabón que, de manera perfectamente ordenada y consecutiva, se encadena en la composición:

\begin{tabular}{|l|l|}
\hline Poema & Análisis \\
\hline $\begin{array}{l}\text { Habitante de los anchos portales } \\
\text { donde el laurel de la sombra oculta el arpa de la araña, }\end{array}$ & Introducción de Apolo. \\
\hline $\begin{array}{l}\text { ¡El silencio dictado por tu mano, } \\
\text { apolíneas } \\
\text { la línea entre tus labios sostenida, }\end{array}$ & Recreación de las formas. \\
\hline $\begin{array}{l}\text { ¡Eterna, eternamente un universo a imagen tuya! } \\
\text { apolíneas } \\
\text { Con la frente a la altura de tu plinto, }\end{array}$ & Devoción a las formas. \\
\hline
\end{tabular}




\begin{tabular}{|l|l|}
\hline $\begin{array}{l}\text { Beso a mi voz, que expresa tu mandato, } \\
\text { la suelto y voy hacia ti, como paloma } \\
\text { obediente en su vuelo }\end{array}$ & Devoción sumisa. \\
\hline $\begin{array}{l}\text { Sobre mí, solo eterno } \\
\text { tu mandato de luz, Verdad y Forma. }\end{array}$ & $\begin{array}{l}\text { Resolución explícita } \\
\text { (el eterno mandato sobre el } \\
\text { yo poético). }\end{array}$ \\
\hline
\end{tabular}

De esta forma, el poema no sólo nutre su estética en las formas de Apolo, sino que, de igual forma, se alimenta de sintagmas verbales que alaban de manera manifiesta las formas apolíneas, además de construirse sobre un orden estrófico-temático también apolíneo (en tanto que deliberada y perfectamente encadenado) mostrando, así, una perfecta combinación entre el dios griego como origen del poema y la filosofía apolínea propia de esta divinidad como construcción explícita (sintagmas verbales) e implícita (orden estrófico) del poema en cuestión. En este sentido, Chacel muestra una defensa del ideario apolíneo a través del poema e, implícitamente, rehúye de lo dionisiaco. En palabras de Isabel Paraíso de Leal:

La exploración de la poesía chaceliana me ha conducido inevitablemente hasta dos conceptos emblemáticos: lo apolíneo y lo dionisíaco. Esta famosa dicotomía de Nietzsche sintetiza, creo, la lucha antagónica que subyace en la poesía de Rosa Chacel, al tiempo que estructura su voz poética y le confiere individualidad [...] Frente al sereno y razonable Apolo, Dionisos es la desmesura, el júbilo doliente, la transfiguración (1994, p. 33).

El poema viene a recoger a través de sus versos la idea de la belleza de la mesura y la belleza del orden (o, lo que es lo mismo, la belleza de lo apolíneo), con lo cual se desecha la posibilidad de hallar beldad en la desmesura o en el desorden, es decir, la reafirmación de la ausencia de belleza en lo dionisiaco. De cualquier modo, nos es conveniente poner en relieve que si bien la división que practicamos sobre los conceptos de lo apolíneo y lo dionisiaco con el propósito de mostrar la adhesión de la lírica chaceliana al trazo apolíneo, lo cierto es que ambos conceptos no parecían residir de manera independiente en su origen clásico, ya que según las reflexiones nietzscheanas recogidas en su $E l$ nacimiento de la tragedia en el espíritu de la música (escrito entre 1871-1872), la existencia de uno suponía la existencia contenida del otro: 
Así, pues, lo dionisiaco propiamente griego es impensable sin la contención de lo apolíneo. Esta contención apolínea de lo dionisiaco, este hacer griego al desbordamiento bárbaro se logra mediante la incrustación de lo apolíneo en lo dionisiaco. Lo dionisiaco griego sólo tiene sentido limitado e invadido por lo apolíneo, y lo apolíneo mismo se conoce como fundamentado -como recuerdo de su propio origen- por lo dionisiaco. Si en tanto fundamentado en él, Apolo no puede vivir sin Dioniso, tampoco Dioniso griego puede vivir sin Apolo (Grave, 1998, p. 50).

El título del poema, como paratexto vital para la comprensión del mismo, muestra en sí una clara alabanza a la mesura y el orden correspondiente al mito de Apolo (en tanto que "Apolo") o, al menos, nos ofrece un horizonte de expectativas que, efectivamente, se cumple con la lectura del poema: la belleza residente en la línea apolínea. Así pues, de manera implícita, sí podemos hacer nuestras las teorías de Nietzsche sobre el origen de la tragedia griega ya que, si en "Apolo" se prioriza la belleza apolínea y se ensalza el valor de la beldad en la mesura, se reniega del desorden y de las formas desproporcionadas propias de Dioniso (formas contenidas en el poema): las teorías de Nietzsche, por lo tanto, bien podrían dar respuesta a la composición lírica de Rosa Chacel.

Es por todo ello por lo que hemos decidido titular el estudio dedicado a la génesis y estética de "Apolo" como clasicismo apolíneo ya que, como ya hemos apuntado con anterioridad, el poema se crea (y recrea) sobre materiales clasicistas (la divinidad mitológica correspondiente a Apolo -a partir de su manifestación escultural-) y nutre su estética en los rasgos apolíneos propios del dios homónimo (de manera sintagmática y estrófico-temática) en una perfecta combinación de materiales líricos (Apolo y sus particularidades físicas) e ideario propio de los mismos materiales (la belleza del rasgo apolíneo propio, efectivamente, de Apolo). Material, forma e ideología actúan, pues, como un único elemento que se retroalimenta en el poema, dando así buen detalle de la astucia artística, a la par que del acervo cultural, de Rosa Chacel como creadora de poemas.

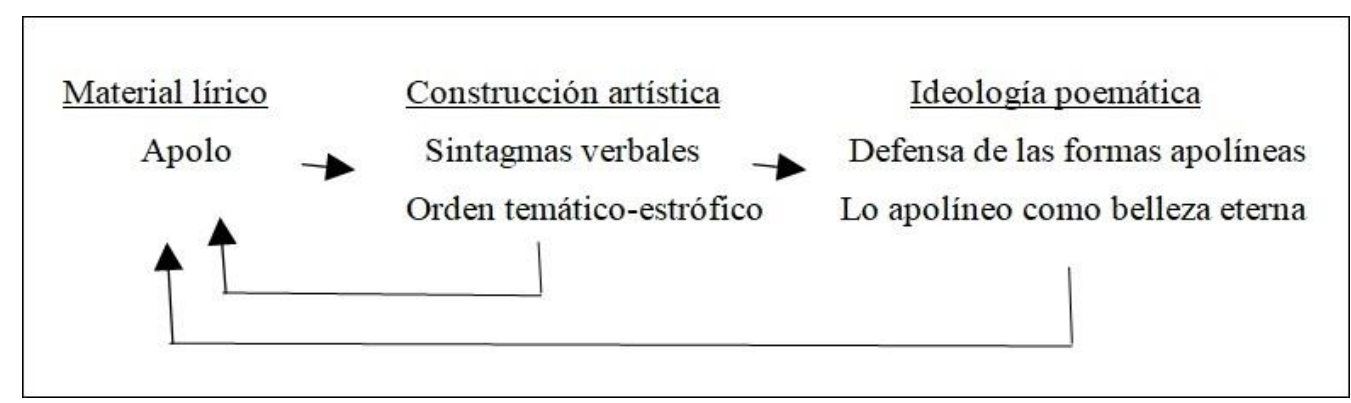




\section{Conclusiones}

El estudio de la génesis del poema de Chacel escogido por sus particularidades y su cuidada estética nos ha llevado a profundizar en temas tan dispares como las teorías nietzscheanas sobre la existencia de lo apolíneo y lo dionisiaco, el culturalismo intelectual de la producción poética española coetánea a Rosa Chacel o la influencia europea a través de las Vanguardias en los autores de la Generación del 27, “primera generación española con miras europeas ” (Lanz Rivera, 2007, 233). Por otro lado, y en referencia a la investigación que hemos llevado a cabo en este artículo, creemos que el caso de la producción poética de Rosa Chacel presenta en sí un acervo cultural extraordinario basado en la fuente, particularmente, de materiales líricos que sitúan su origen en la antigua Grecia y sus deidades afirmación que queda evidente ya desde el título que antecede al poema: “Apolo"-.

En cualquier caso, hemos apreciado cómo nuestra teoría acerca de la existencia de una transmutación de materiales artísticos en el poema analizado, o significantes, que hallan su precedencia en artes plásticas heterogéneas (la escultura de Apolo, de forma más exhaustiva) para pasar a convertirse en palabra lírica y, con ello, en literatura, es decir, otro tipo de material artístico. La idea resulta ahora nada desdeñable en tanto que la propia autora era una fiel apasionada de las artes esculturales y pictóricas, tal y como se ha citado a lo largo del artículo, a las que incluso dedicó su estudio durante su época adolescente y, particularmente, la impresión que le produjo la contemplación de la escultura a quien, tal y como ella misma alude, le dedicó el poema. Por lo tanto, si bien la autora vallisoletana cambió la escultura y la pintura por la literatura, hemos podido comprobar cómo en el fondo (en el significado según la distinción semiótica y/o saussurreana) siempre se mantuvo leal a su primer amor artístico, ya que fueron estos materiales plásticos los que conformaron el universo artístico de su producción poético-literaria. El caso de "Apolo" invita así a reformular las aproximaciones al poema que han tenido lugar hasta la fecha, abriendo el abanico de interpretaciones acerca del vínculo entre estética y génesis en cuanto a la propia creación lírica chaceliana. De igual modo, el artículo ha demostrado que la -en ocasiones- férrea clasificación de los materiales artísticos (si bien dicha clasificación es de gran utilidad para determinados fines, como los docentes) son desdibujados en el proceso de construcción lírica a través de transmutaciones de los materiales, lo que, en última instancia, pone en relieve la importancia de la transversalidad de disciplinas en cuanto al análisis e investigación del arte en todas sus formas posibles. 


\section{Referencias bibliográficas}

CHACEL, Rosa (1992). Poesía (1931-1991). Tusquets: Barcelona

CONTE, Rafael (1993). “Rosa Chacel y las Vanguardias” en LATRE, María Pilar Martínez, Actas del congreso en homenaje a Rosa Chacel: ponencias y comunicaciones. Universidad de la Rioja: Logroño, pág. 22.

GRAVE, Crescenciano (1998). El pensar trágico: Un ensayo sobre Nietzsche. UNAM: México.

GRIMAL, Pierre (2008 [1951]). Diccionario de mitología griega y romana. Grupo Planeta: Barcelona. JANÉS, Clara (2010). María Zambrano. Desde la sombra llameante. Ediciones Siruela: Madrid.

LANZ RIVERA, Juan José (2007). "La poesía española ante la feria” en La poesía durante la transición y la generación de la democracia” en PASTOR, Juan (ed.). Devenir Ensayo: Madrid, pág. 233.

OSBORNE, Raquel (1987). "Simmel y la "cultura femenina" (las múltiples lecturas de unos viejos textos)” en Reis: Revista española de investigaciones sociológicas, № 40, págs. 97-112.

PARAÍSO DE LEAL, Isabel (1994). "Lo apolíneo y lo dionisiaco en la poesía de Rosa Chacel” en LATRE, María Pilar, Actas del congreso en homenaje a Rosa Chacel: ponencias y comunicaciones. Universidad de la Rioja: Logroño, págs. 33 y 43.

PÉREZ CHACEL, Carlos y Antonio Piedra (2004). Rosa Chacel. Obra completa: Autobiografías. Fundación Jorge Guillén: Valladolid.

QUESADA MARCO, Sebastián (1997). Diccionario de civilización y culturas españolas. Ediciones Istmo: Madrid. 